\title{
Diffraction Peak Broadening Anisotropy of Shock Loaded Uranium
}

\author{
Alexander Shestakov ${ }^{1, *}$, and Dmitry Belyaev ${ }^{1}$ \\ Federal State Unitary Enterprise «Russian Federal Nuclear Center - Zababakhin All-Russia Research \\ Institute of Technical Physics», 456770, Vasilieva str. 13, Snezhinsk, Russia
}

\begin{abstract}
Analysis of angular dependences of the diffraction peak broadening observed in $\alpha$-uranium in as-received state, after loading by converging spherical shock wave, low-speed uniaxial deformation, and annealing at $850^{\circ} \mathrm{C}$ has been presented in this work. Broadening anisotropy identical for all the states investigated has been demonstrated. The authors have attempted to explain the phenomena by Young's modulus anisotropy of uranium or the crystallite orientation relative to the load applied under plastic deformation.
\end{abstract}

\section{Introduction}

Any deformation leads to broadening of the diffraction peaks in X-ray patterns $[1,2]$. Dependence of their broadening on the diffraction angle conveys information about the mechanisms and degree of the material deformation. Analysis of the angular dependence in $\mathrm{X}$-ray patterns allowed the authors to reveal a regular nonmonotonicity in the material after various effects. Figure 1 shows a typical example of nonmonotonicity. Increase in the diffraction angle results in a sharp decrease of peak (043) width which is followed by a sharp increase observed in peak (150). The changes are far beyond as compared to an error in FWHM determination.

A phenomena like this, in case of $\delta$-phase Pu-Ga alloy is attributed to Young's modulus anisotropy and microstresses in the lattice [3]. Young's modulus anisotropy was taken into account when calculating microstresses in uranium lattice [4]. This work is focused on an attempt to explain the observed diffraction peak broadening anisotropy in uranium after various effects.

\section{Devices and techniques}

$\mathrm{X}$-ray diffraction measurements were made under $\mathrm{CuK}_{\alpha}$ radiation using an X-ray diffractometer with the Bragg-Brentano focusing. The analyzed layer thickness measured in uranium exposed to $\mathrm{CuK}_{\alpha}$ radiation was about a micrometer. This posed high-level requirements to preparation of the surface for investigation. The cold worked layer was removed by electrochemical polishing. Thickness of the removed material was obtained from a decrease in the specimen mass. More than $100 \mu \mathrm{m}$ were removed from the

\footnotetext{
*Corresponding author: a.e.shestakov@vniitf.ru
} 
specimens to assure removal of the cold-worked layer even after the specimens were cut in pieces using an abrasive disc.

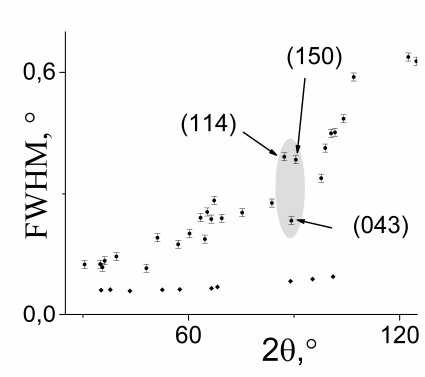

a)

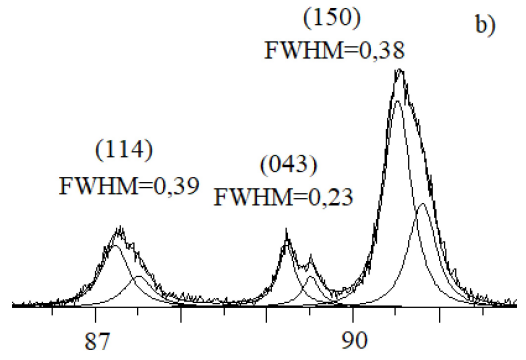

Fig. 1. a) Full width on half maximum of diffraction peaks versus diffraction angle for uranium loaded by converging spherical shock wave. Below is the instrumental function. b) Diffraction spectrum region (Fig. 1a at the left shows highlighted dots corresponding to these peaks).

Characteristic copper radiation. Miller indices and full width on half maximum values are given.

The corundum standard NIST1976 was utilized to design the instrumentation function Physical broadening was calculated by means of the formula:

$$
\beta=F W H M-b
$$

where: $F W H M$ is full width on half maximum of the diffraction peak; $\beta$ is physical broadening; $b$ is instrumental broadening.

The Williamson-Hall method was used to analyze the angular dependence of broadening. [5]. Physical broadening was presented in terms of:

$$
\beta=\lambda \cdot(D \cdot \cos (\theta))^{-1}-4 \varepsilon \cdot \operatorname{tg}(\theta)
$$

where: $D$ is the crystalline size; $\lambda$ is the wavelength of used radiation; $\varepsilon$ is the value of microdistortions.

Metallographic study was carried out employing light microscopy on the surfaces of the specimens prepared by abrasing machining and polishing with the diamond paste of different granularity. Electropolishing, as a final operation, was used to remove the cold worked layer. The structure contrast was enhanced by means of chemical, electrochemical or argon ion beam etching. Vickers microhardness testing was performed under 50-gf.

Young's moduli for various crystallographic directions were calculated as in Ref. [4]. Calculation based on Fischer data is given in Ref. [6].

Direction [023] is the hardest one $\left(E_{023}=287.7 \mathrm{GPa}\right)$, direction [010] is the softest one $\left(E_{010}=148.6 \mathrm{GPa}\right)$. Figure 2a shows the result in the form of a stereographic projection on plane (001). Squares stand for the hard directions corresponding to 287.7 $\mathrm{GPa}<E<241.3 \mathrm{GPa}$, rhombi stand for the soft ones corresponding to 148.7 $\mathrm{GPa}<E<195 \mathrm{GPa}$. The remaining directions are denoted by circles.

\section{Initial state of material}

All the specimens under study were made of coarse-grained technical-grade uranium. Carbon, as a major impurity, was present in the material in the form of carbides (Fig. 2b). Figure 3a shows a region in X-ray pattern demonstrating material in its initial state. This region is bearing the uranium carbide diffraction peak. The material grain-size was equal to 130-3000 $\mu \mathrm{m}$. The grain shape was non-equiaxial. The metal grains contained twins, more than two twinning systems. The material microhardness was equal to $311 \mathrm{~kg} / \mathrm{mm}^{2}$ within a wide statistical dispersion of $46 \mathrm{~kg} / \mathrm{mm}^{2}$. The dispersion is associated with mechanical anisotropy of individual uranium crystals [7]. 
Figure $3 \mathrm{~b}$ illustrates Williamson-Hall plot for this specimen. The effective value and the lattice microdistortion values $\varepsilon_{\text {eff }}$ are $0.05 \%$. The figure clearly shows that the dispersion of values is well over the error. Diffraction peaks (020) and (040) are above the line and peaks (021) and (042) are below the line. All of this is indicative of a regular dispersion and can be attributed to crystallographic directions. The authors in Ref. [4] associate this effect with Young's modulus anisotropy. Figure 2c shows the hard directions indicated by squares and the soft ones indicated by rhombi. It is obvious that not all of the hard reflexes are below and not all of the soft ones are above the effective values. The hard reflex (223) is much wider than the soft one (241). The soft reflex (150) is the widest but peak (043) belonging to an average-rigidity group is the narrowest. An attempt to split broadening into corresponding Young's moduli that was made in much the same manner as it had been done by the authors in Ref. [4] did not contribute to a lower dispersion.
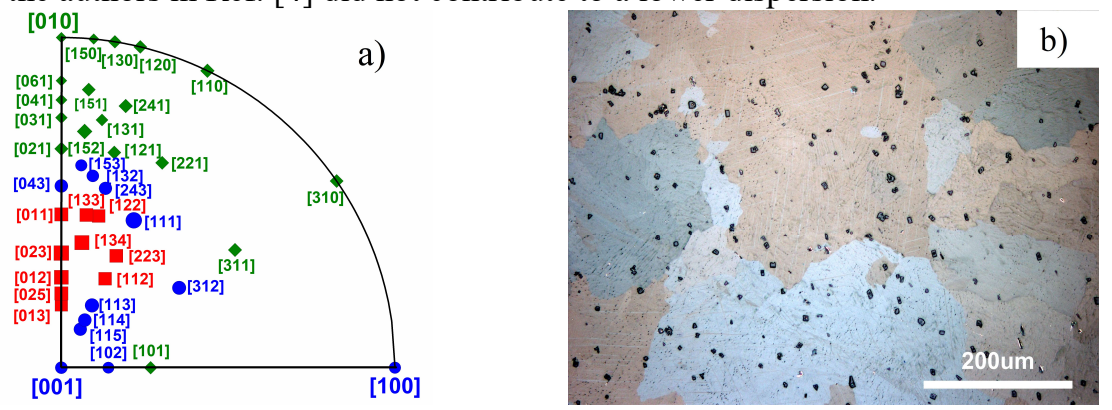

Fig. 2. a) Stereographic projection of crystallographic directions in $\alpha$-U lattice. Hard (with a big value of Young's module) directions are denoted by squares, soft directions - by rhombi, average directions - by circles. b) Material structure in its initial state. Non-equiaxial coarse $\alpha$-grains, subgrains; two-system twins, carbide inclusions
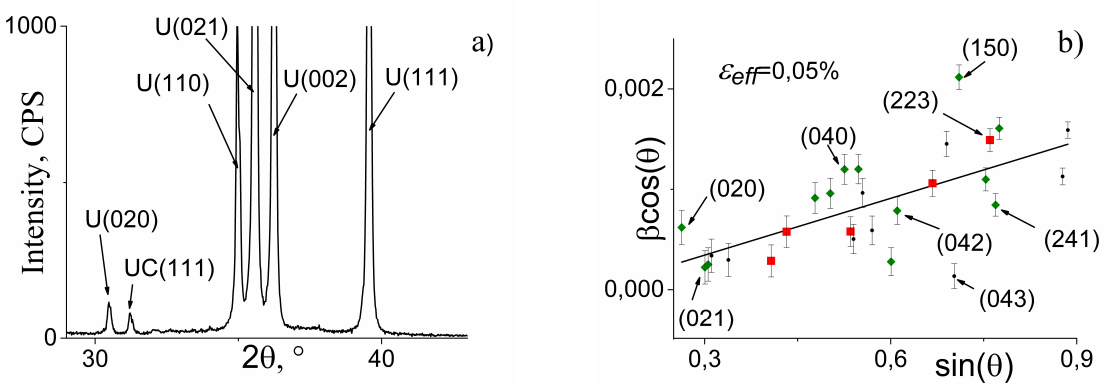

Fig. 3. Initial state of material. a) Diffraction spectrum region. Characteristic copper radiation. Lines of $\alpha$-uranium and uranium carbide are noted. b) Williamson-Hall plot. Dispersion of points is much greater than the error estimate. Hard lines are denoted by squares, soft lines — by rhombi, and average lines — by circles.

\section{Material after shock wave loading}

A thick-walled shell was recovered after loading by converging spherical shock wave. Meanwhile, the external and internal radii reduced and the shell thickness increased. From the estimate, it was inferred that the material near the outer surface had been subjected to $20 \%$ tensile strain along its radius. Furthermore, the entire deformation process occurred at elevated temperatures but in $\alpha$-phase. Microhardness of the material increased up to $\sim 375 \ldots 400 \mathrm{~kg} / \mathrm{mm}^{2}$ as compared to its initial state. The material near the internal surface underwent the resultant tensile strain above $100 \%$. The phase analysis showed that the material was in $\alpha$-phase but the results of light microscopy unambiguously demonstrated 
that it had gone through phase transformations. Figure 4a shows the material structure near the internal surface. The recrystallized grain size reduced to $40-50 \mu \mathrm{m}$. The grains are equiaxial, the twins are of a small-scale density. Microhardness within the internal layers dropped to $250-300 \mathrm{~kg} / \mathrm{mm}^{2}$ [8].

A spherical shell after shock wave loading was cut into two parts along the meridian. Thereafter, a segment was cut out by two runs of a circular saw. The resultant segment was used to cut a small slug (Fig. 4 b) $\sim 5 \times 5-\mathrm{mm}$ in size. The north end of this slug was cut into transverse specimens
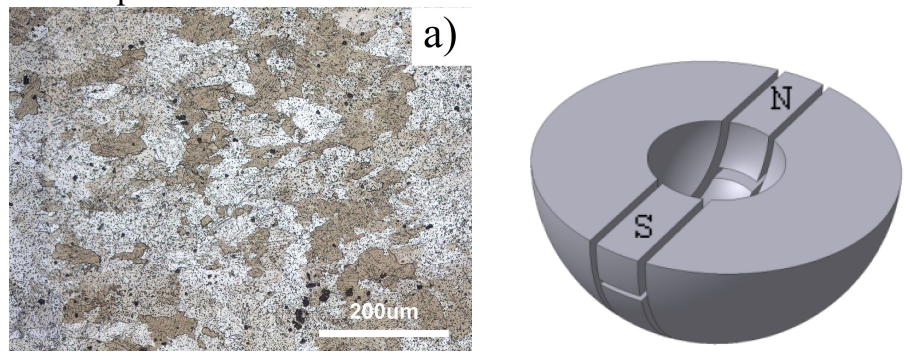

b)

Fig. 4. a) Material structure near the internal structure. Equiaxial grains with the twins of a smallscale density. Grains $40-50 \mu \mathrm{m}$ in size. b) Cutting layout (recovered shell cut into parts to prepare specimens for X-ray diffraction analysis).

Williamson-Hall plot showed that the effective value of the lattice distortion within the outer layers $\varepsilon_{\text {eff }}=0.13 \%$ (Fig. 5a) is larger than that of the internal layers $\varepsilon_{\text {eff }}=0.08 \%$ (Fig. $5 b)$. Both values are larger than the values obtained in the initial state and thus they are in agreement with the obtained values of microhardness. One and the same scale was used for both figures to demonstrate the difference between the slopes of lines.

Dispersion in both plots is of a regular character. Diffraction peaks (020) and (040) are above the lines and peaks (021) and (042) are below. Hard reflex (223) is wider than the soft one (241). Soft reflex (150) is the widest and peak (043) that belongs to an averagerigidity group is one of the narrowest.
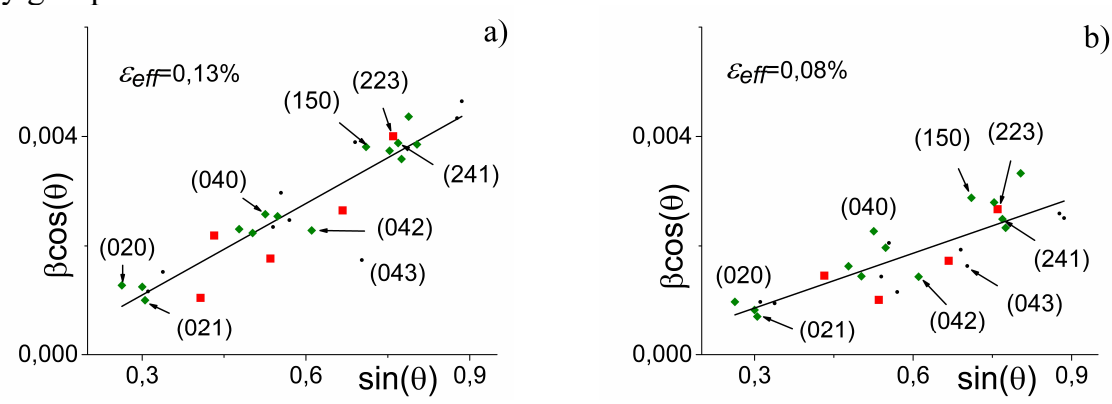

Fig. 5. a) Williamson-Hall plot for shock loaded material. Relative deformation of about $20 \%$ in $\alpha$ phase. Typical mutual arrangement of points is noted. b) Williamson-Hall plot for shock loaded material that underwent phase recrystallization. Typical mutual arrangement of points is noted.

A hypothesis for isotropic stress state and anisotropic Young's modulus falls short of explaining the observed phenomena. Assume that the stress is not isotropic. When the load is being applied, not all the crystallites begin to undergo simultaneous plastic deformation. The crystallites whose slip planes are at an angle of $45^{\circ}$ to the applied load direction (Schmid factor is 0.5 ) will be the first to undergo deformation and this deformation will be the highest. To test this hypothesis, maximum Schmid factors correlating the applied load with shear stresses of various slide systems in uranium were calculated. Table 1 shows the 
obtained values for some of the crystallographic directions and the principal slip systems [9-10].

It is apparent that for wide reflex (150) the factor is small and for narrow reflex (043) the factor has nearly a maximum value. A similar situation occurs with reflexes (020) and (021). In this way, various orientations of the deformed crystallites relative to the applied load cannot be an explanation for the observed diffraction peak broadening anisotropy.

Table 1. Schmid factor for some of the directions and slip planes

\begin{tabular}{|c|c|c|c|c|}
\hline \multirow{2}{*}{ Slip planes } & \multicolumn{4}{|c|}{ Crystallographic directions of applied loading } \\
\cline { 2 - 5 } & {$[150]$} & {$[043]$} & {$[010]$} & {$[021]$} \\
\hline$(110)$ & 0.30 & 0.49 & 0.40 & 0.46 \\
\hline$(010)$ & 0.09 & 0.45 & 0 & 0.36 \\
\hline$(001)$ & 0 & 0.45 & 0 & 0.36 \\
\hline
\end{tabular}

\section{Material after low-speed deformation and annealing}

A 2-mm thick specimen was plastically deformed by press at room temperature. The degree of deformation was about $20 \%$. This was much similar to the material deformation within the outer layers of the recovered shell. To eliminate the surface effect action, about $0.5 \mathrm{~mm}$ was removed from the material surface using the abrasive materials and $0.1 \mathrm{~mm}$ by electropolishing during the specimen preparation. The specimen surface was perpendicular to the deformation direction. Figure $6 \mathrm{~b}$ shows Williamson-Hall plot for the deformed material. The number of points is smaller as compared to the previous figures because the lines in X-ray patterns are very wide and low in terms of intensity (Figure 6a). In this case, the effective value of microdistortion $\varepsilon_{e f f}=0.18 \%$ is larger than that of the material subjected to shock wave deformation.

From this figure we notice that hard reflex (223) is wider as compared to soft reflex (241) and peak (150) is the widest with the least Schmid factor.
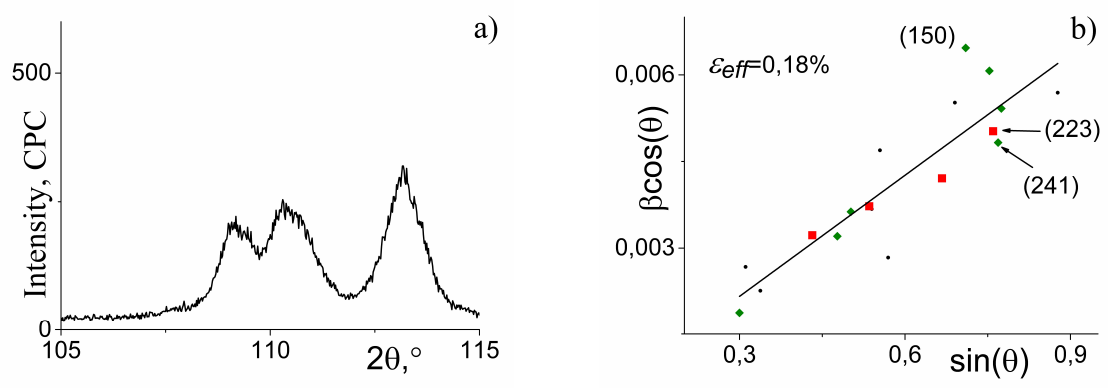

Fig. 6. Material subjected to uniaxial deformation at 20\%. a) X-ray pattern region. Characteristic copper radiation. Diffraction peaks are broadened and their intensity is small-scale. b) WilliamsonHall plot. Typical mutual arrangement of points is noticed.

Material after $20 \%$ uniaxial deformation was annealed at $850^{\circ} \mathrm{C}$ for half an hour. The specimen was cooled with the furnace. The surface was prepared for measurements in a manner identical to that for the deformed material. The diffraction peaks became very narrow and the effective value of microdistortion $\varepsilon_{e f f}=0.02 \%$ was smaller as compared to the material in its initial state.

Figure 7 shows a region in X-ray pattern and Williamson-Hall plot. The dispersion character is similar than all of the previous ones. The hard line (223) is wider than the soft one (241) and peak (150) is the widest with the least Schmid factor. Line (043) is one of the narrowest even though it is not a hard one. 

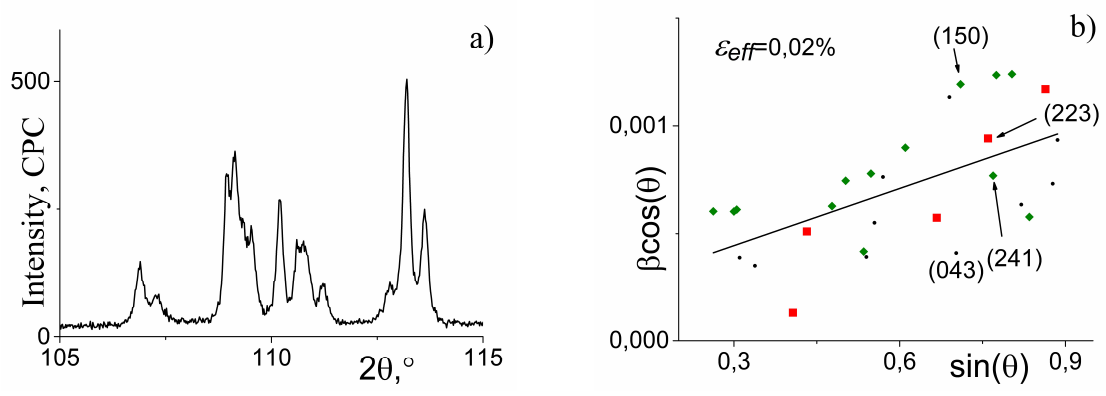

Fig. 7. Material after uniaxial deformation at $20 \%$ and annealing at $850{ }^{\circ} \mathrm{C}$. a) X-ray pattern region. Characteristic copper radiation. Diffraction peaks are very narrow. b) Williamson-Hall plot. Typical mutual arrangement of points is noticed.

\section{Conclusion}

It has been demonstrated that the diffraction peak broadening anisotropy of uranium loaded by converging spherical shock wave occurred within the layers that were deformed in $\alpha$ phase as well as within the layers that underwent phase transformations. A similar anisotropy was detected resulting from uniaxial low-speed deformation and deformation and annealing at $850^{\circ} \mathrm{C}$.

A hypothesis for isotropic stress state and anisotropic Young's modulus falls short of explaining the observed phenomena. This effect cannot be described in terms of deformation associated with the crystallite orientation relative to the applied load.

The diffraction peak anisotropy in uranium cannot be described unless both of the abovementioned reasons, as well as potential anisotropic grinding of crystallites during the twinning process are considered.

\section{References}

1. R. Krishnan, V.S. Arunachalam, M.K. Asundit, Acta Metallurgica, 10, 75-78 (1962)

2. A.V. Pavlenko, A.E.Shestakov, A.R.Nurgaleev, D.N.Kazakov, , Feature in accumulation of microdefects in copper under shock-wave loading, in Proceedings of the $8^{\text {th }}$ International Conference on Mechanical and Physical Behaviour of Materials under Dynamic Loading, EURODYMAT2006, Dijon, France (2006)

3. J. Zhang, B. Clausen, A.I. Smith, F.J. Freibert, S.C. Vogel, D.W. Brown, JNM, 517, 152-156 (2019)

4. J.E. Sutcliffe, J.R. Pertherbridge, T. Cartwright, R. Springell, T.B. Scott, J.E. Darnbrough, Materials Characterization, 158,109968 (2019)

5. G.K. Williamson, W.M. Hall, Acta Metall. 1, 22-31 (1953)

6. E.S. Fisher, JNM, 18, 39-54 (1966)

7. Yu.N. Sokursky, Ya.M. Sterlin, E.A. Fedorchenko. Uranium and its alloys. - M.: "Atomizdat", 1971. 446 p.

8. D.A. Belyaev, Yu.N. Zuev, E.A. Kozlov, I.V. Podgornova, I.L. Svyatov, Application of Color Mapping Method to Metallographic Study of the Samples after Dynamic Loading. Abstracts of IX International Ural Seminar on Radiation Damage Physics of Metals and Alloys, Kyshtym, Russia (2011)

9. L.T. Lloyd, H.H. Chiswik, J. Metals, 7, 1206-1214 (1955)

10. S.L. Daniel at al., J. Nucl. Mater., 26, 319-324 (1968) 